\title{
ATLAS LAr Calorimeter Performance in LHC Run 2
}

\section{Mahon ${ }^{1}$ on behalf of the ATLAS Liquid Argon Calorimeter Group}

\author{
${ }^{1}$ Nevis Laboratories, Columbia University \\ E-mail: devin.mahon@cern.ch
}

\begin{abstract}
AвSTRACT: Liquid argon (LAr) sampling calorimeters are employed by ATLAS for all electromagnetic calorimetry in the pseudorapidity region $|\eta|<3.2$, and for hadronic and forward calorimetry in the region from $|\eta|=1.5$ to $|\eta|=4.9$. In the first LHC run a total luminosity of $27 \mathrm{fb}^{-1}$ has been collected at center-of-mass energies of 7-8 TeV. After detector consolidation during a long shutdown, Run 2 started in 2015 and $147 \mathrm{fb}^{-1}$ of data at a center-of-mass energy of $13 \mathrm{TeV}$ have been recorded. In order to realize the level-1 trigger acceptance rate of $100 \mathrm{kHz}$ in Run 2 data taking, the number of read-out samples recorded and used for the energy and the time measurement has been modified from five to four while keeping the expected performance. The well calibrated and highly granular LAr calorimeter reached its design values both in energy measurement as well as in direction resolution. This contribution gives an overview of the detector operation, hardware improvements, changes in the monitoring and data quality procedures, to cope with increased pileup, as well as the achieved performance, including the calibration and stability of the electromagnetic scale, noise level, response uniformity and time resolution.
\end{abstract}

Keywords: Calorimeters, Performance of High Energy Physics Detectors, Noble liquid detectors (scintillation, ionization, double-phase) 


\section{Contents}

1 Introduction 1

1.1 ATLAS and the LHC 1

1.2 ATLAS LAr Calorimeter 1

1.3 Signal Measurement and Readout 2

2 Data Quality 3

2.1 Defect Rejection 3

2.2 Veto Rejection 4

3 Performance and Operation Studies $\quad 4$

3.1 Purity Stability 5

3.2 Pulse Shapes 5

3.3 Energy Resolution 6

$\begin{array}{lll}3.4 & \text { Timing } & 7\end{array}$

4 Phase I Upgrade $\quad 7$

5 Conclusions $\quad 8$

\section{Introduction}

\subsection{ATLAS and the LHC}

CERN's Large Hadron Collider (LHC) is the world's highest energy particle collider with both proton-proton and heavy ion collision modes, a center-of-mass energy up to $\sqrt{s}=13 \mathrm{TeV}$, a peak instantaneous luminosity of $2.1 \times 10^{34} \mathrm{~cm}^{-2} \mathrm{~s}^{-1}$, and a bunch crossing rate of $40 \mathrm{MHz}$. During proton-proton bunch crossings, multiple collisions may occur-a phenomenon known as pileup. During Run 2, the average pileup was 33.7, but different beam configurations resulted in pileup as high as $\sim 80$. The LHC operated very successfully during Run 2, the 2015-2018 data-taking period, delivering $156 \mathrm{fb}^{-1}$ of data [1].

The ATLAS detector is one of the two general purpose detectors at the LHC. As such, it provides tracking, calorimetry, and muon spectrometry. ATLAS recorded $147 \mathrm{fb}^{-1}$ of data, of which $139 \mathrm{fb}^{-1}$ was considered good for physics, reflecting an overall efficiency of $89 \%[1,2]$.

\subsection{ATLAS LAr Calorimeter}

The ATLAS liquid argon (LAr) calorimeter is a sampling calorimeter consisting of electromagnetic (EM) and hadronic sections with cryogenically-cooled liquid argon as the active medium. Argon is advantageous for calorimetry for many reasons including its linear response, uniformity, stability 
over time, and radiation-tolerance. The LAr calorimeter contains $\sim 182,000$ channels, of which $>99.9 \%$ were operational throughout Run 2 [3].

The EM barrel (EMB) and end-cap (EMEC) calorimeters have lead plates interspaced as the passive material with electrodes arranged in accordion-like structures. This unique structure is selfsupporting and allows for fast readout and full, uniform azimuthal coverage without dead material from structural supports. The coverage in pseudorapidity of the EMB and EMEC calorimeters are $|\eta|<1.475$ and $1.375<|\eta|<3.2$, respectively.

The forward calorimeters (FCal) contain copper and tungsten absorber matrices with rod electrode structures. Liquid argon fills the gaps between these rods and absorber matrices. This section occupies the region $3.1<|\eta|<4.9$.

The hadronic end-cap (HEC) calorimeters are conventional parallel copper plate electrodes occupying the region $1.5<|\eta|<3.2$.

The structures of these detector sections are detailed in Figure 1.

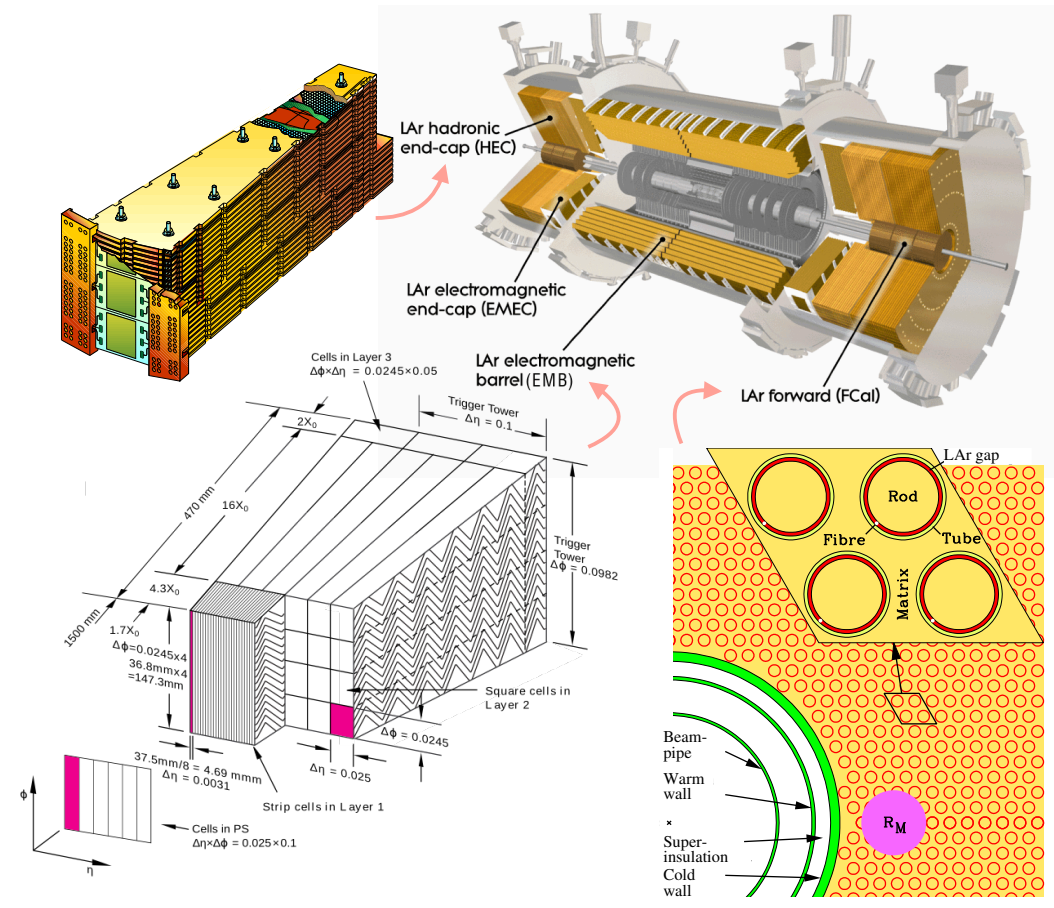

Figure 1: Diagram of the LAr calorimeters, consisting of electromagnetic barrel (EMB) and end-caps (EMEC), forward calorimeters (FCal), and the hadronic end-caps (HEC) [3].

\subsection{Signal Measurement and Readout}

When an incoming electromagnetic particle hits the lead absorber, an electromagnetic shower is produced in the liquid argon, the resulting current from which is then collected by electrodes. The resulting triangular pulse is sent to the on-detector Front-End Boards (FEBs) where it is amplified, split into three overlapping linear gain scales, and shaped. An illustration of the shaped and unshaped ionization pulse is shown in Figure 2. The pulse is then sampled at $40 \mathrm{MHz}$ and stored in an analog buffer awaiting a trigger decision. If triggered, a gain is selected, and four samples are 
digitized and read out via optical fiber. Off-detector, optimal filtering coefficients (OFCs) are used to compute the pulse amplitude (which is proportional to the energy of the incident object) and time [4]. Additionally, a quality factor is computed, which measures the consistency of the pulse with an in-time LAr ionization pulse. This quantity can be used to mitigate the effect of out-of-time pileup, consisting of objects from collisions from other bunch crossings [3].

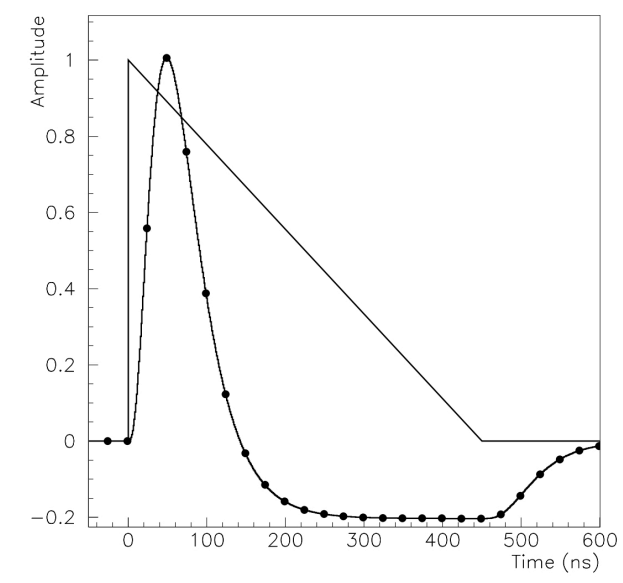

Figure 2: Liquid argon ionization pulse shape as produced in the detector (triangle) and after shaping (curve with dots). The dots are spaced in intervals of $25 \mathrm{~ns}$ corresponding to a frequency of $40 \mathrm{MHz}$, the frequency of successive bunch crossings as well as the readout sampling frequency [3].

\section{Data Quality}

The LAr calorimeters operated extremely efficiently throughout Run 2, achieving a 99.6\% overall data-taking efficiency. This high efficiency can be attributed to very stable system performance as well as a dedicated operations team. Many real-time (online) monitoring tools quickly identify issues, and some automated recovery procedures minimize data loss from common issues [5].

The data inefficiency of $0.4 \%$ attributable to the LAr calorimeters is classified into two categories: defect rejection and veto rejection.

\subsection{Defect Rejection}

Defect rejection refers to rejection of longer blocks of data lasting more than $\sim 1$ minute. The main sources of this type of data loss are noisy channels, noise bursts, coverage, and high voltage (HV) trips [5]. A summary of data losses due to these inefficiencies is summarized in Figure 3(a).

Noisy channels are individual channels that are masked due to noise from pulses incompatible with LAr ionization. Noise bursts are a well-studied coherent noise phenomenon that affects a large fraction of the detector for a short time $(\sim 1 \mu \mathrm{s})$. They are suspected to be induced by unshielded HV cables. Coverage refers to a temporary readout issue in an area of $>512$ cells due to a single hardware failure. HV trips result in unreliable energy corrections. Thus during the sudden voltage drop, data is rejected. When ramping the channel back up, however, such gradual changes in voltage 
can be corrected offline to recover data collected during this time. Additionally, new power supplies were installed before 2016 data-taking, significantly reducing losses from this issue.

\subsection{Veto Rejection}

Veto rejection refers to rejection of data within shorter time periods $(\lesssim 1$ minute). In this case, rejection is done event-by-event. The main sources of this type of data loss are mini noise bursts and data corruption [5]. A summary of data losses due to these inefficiencies is summarized in Figure 3(b).

Like noise bursts, mini noise bursts are coherent noise phenomena, but they affect a smaller region of the detector and for an even shorter amount of time (tens of ns). Improved identification and HV tuning during the data-taking period virtually eliminated their effect by 2018. Data corruption is most commonly due to a desynchronization of one or several FEBs. Data acquired between the desynchronization and the automatic resynchronization are rejected.

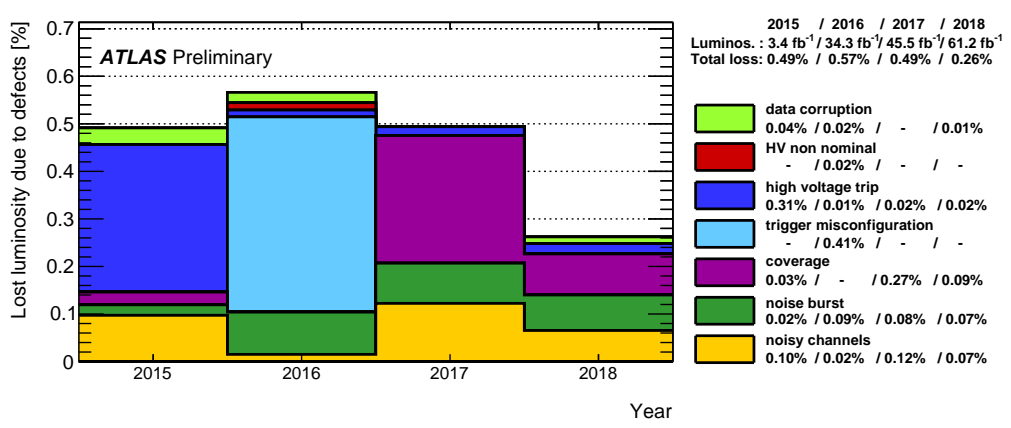

(a)

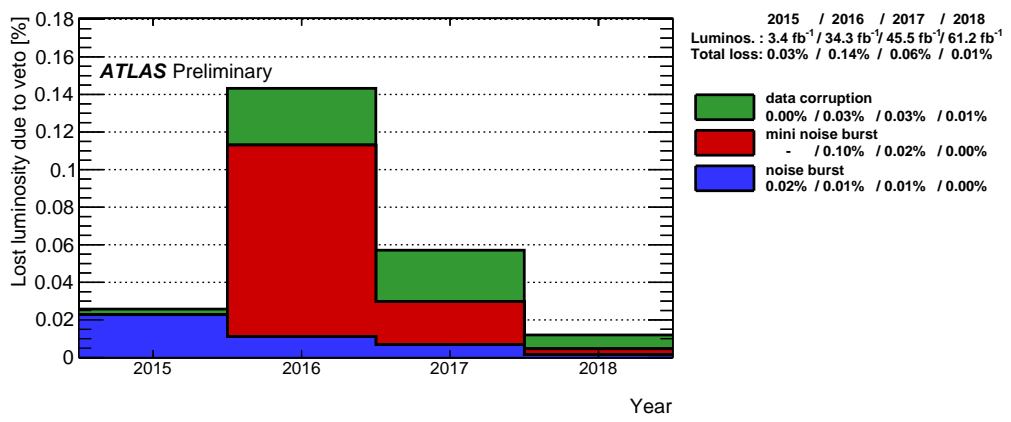

(b)

Figure 3: Summary of LAr calorimeter data inefficiencies in Run 2 by year. (a) shows defect rejection (minute-length issues) and (b) shows veto rejection (sub-minute-length issues) [5].

\section{Performance and Operation Studies}

Throughout Run 2, many studies of performance and detector operation were carried out to evaluate and understand the status of the detector. A few of these studies are presented and discussed below. 


\subsection{Purity Stability}

Maintaining the purity of the liquid argon is essential to ensuring good, stable conditions during data-taking. Impurities in the form of electronegative molecules (primarily $\mathrm{O}_{2}$ and $\mathrm{N}_{2}$ ) in the liquid argon capture signal electrons, preventing them from being collected at the electrodes and thus reducing the signal-to-noise ratio. In order to monitor these impurities, $\alpha$ - and $\beta$-source chambers located in the cryostats measure the electron lifetime in the liquid argon. Additionally, laser chambers in contact with the liquid argon measure both the electron lifetime and drift velocity and provide calibration for the source chambers [3]. Impurity measurements during collisions are excluded due to signals from radiation in the ionization chambers.

As shown in Figure 4, measurements taken throughout Run 2 demonstrate that the impurity remained stable for the end-caps and decreased for the barrel. All measurements were below 0.25 ppm (measured in oxygen-equivalent), well within the design specifications. A clear explanation for the decrease in the barrel is unknown. However, changes in input parameters such as temperature and high voltage of the ionization chambers are excluded by dedicated studies [6].

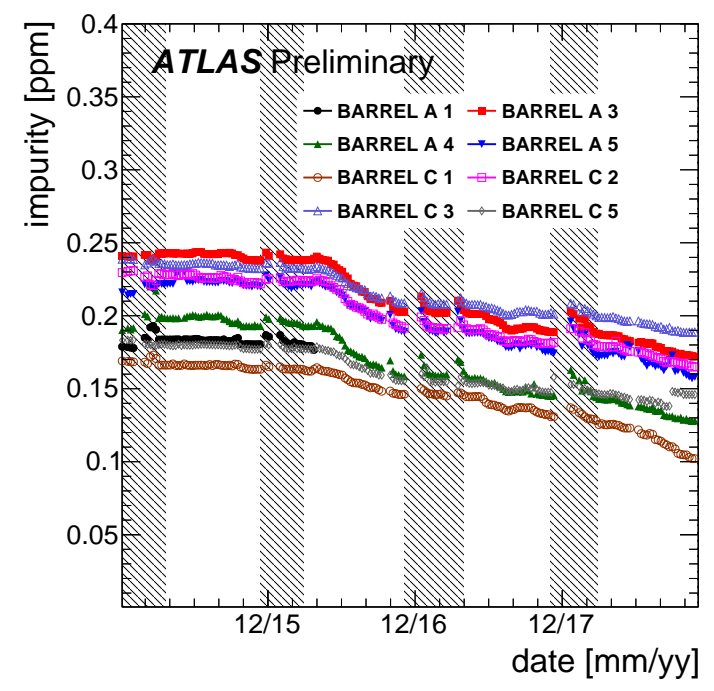

(a)

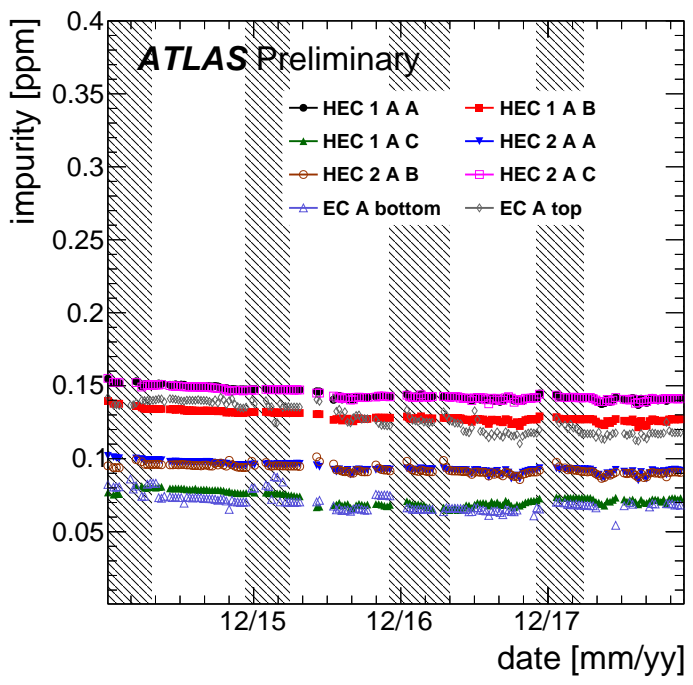

(b)

Figure 4: Mean purity values measured in oxygen-equivalent during Run 2 for (a) the barrel and (b) the end-caps [6].

\subsection{Pulse Shapes}

Precise knowledge of the liquid argon ionization pulse shape is important for several reasons. First, it reduces the uncertainties on the shape corrections in the energy computation. Second, it serves as a cross-check on the liquid argon purity by looking for distortions in the tail of the pulse caused by captured electrons. And thirdly, it improves the so-called baseline correction for the LHC bunch structure. For hypothetically infinite bunch trains, the bipolar pulse shape cancels inand out-of-time pileup contributions to the energy computation. But of course, bunch trains are finite in length, and additionally, the structure of the bunches can change configuration during the 
data-taking period, causing additional variations. By precisely measuring the pulse shape in various bunch configurations, the resulting energy shift can be understood, and a pedestal correction can be applied to mitigate its effects. For a typical EM cluster and typical pileup, the maximum baseline shift in transverse energy due to this effect is $\sim 800 \mathrm{MeV}$. After correction from precise knowledge of the pulse shapes, this shift is reduced to $<30 \mathrm{MeV}$ [5].

During Run 2, several dedicated pulse shape measurements were taken during special runs. During a 2016 special run, four samples were recorded with a random trigger around a single colliding bunch. In 2017 and 2018, special runs with 32 samples were taken, shifting the readout in steps of $\sim 3 \mathrm{~ns}$. With the data from these runs, the ionization pulse shape is now sampled precisely at 256 points in various detector regions.

A sample plot of the pulse shape measurements is shown in Figure 5(a), and a plot of the shift of the transverse energy versus the position in the bunch train can be found in Figure 5(b).



(a)

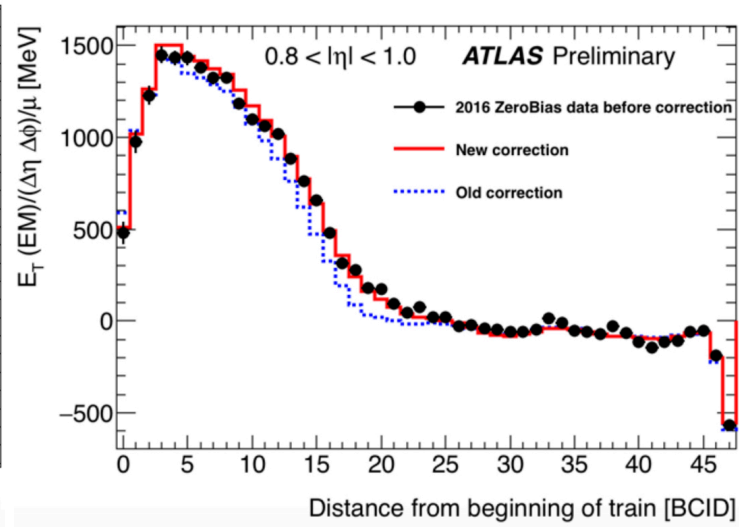

(b)

Figure 5: (a) A typical ionization pulse shape in the barrel presampler in the region $0.8<|\eta|<0.9$. (b) Average transverse energy per unit $\Delta \eta \Delta \phi$ per unit pileup $(\mu)$ versus the distance from the beginning of the bunch train (each unit of distance is $25 \mathrm{~ns}$ ) in the region $0.8<|\eta|<1$.0. Data from special runs in 2016 are in black. The blue line shows the old baseline correction, and the red line shows the new correction based on improved pulse shape knowledge from these special runs [5].

\subsection{Energy Resolution}

Several studies of the LAr calorimeter energy resolution and its modeling in Monte Carlo (MC) simulation were carried out.

The energy scale and energy resolution of MC simulation are calibrated by comparing $Z \rightarrow e e$ data and simulation. The resulting corrections are then verified with $Z \rightarrow e e \gamma$ and $Z \rightarrow \mu \mu \gamma$ data. As shown in Figure 6(a), the residual scale factors after applying the corrections indicate that the calibration results in an agreement with precision $\lesssim 0.5 \%$ [7].

Higher pileup $(\mu)$ increases noise and thus worsens the energy resolution $(\propto \sqrt{\mu})$. The stability of the calorimeter performance as a function of pileup is especially relevant looking forward to Run 3 and beyond when there will be a much higher pileup environment. Figure 6(b) shows the 
variation of the $Z$ mass with pileup in $Z \rightarrow e e$ data and MC simulation, and it demonstrates a stability within $\sim 0.05 \%$ from $\mu=10$ to 70 [7].



(a)

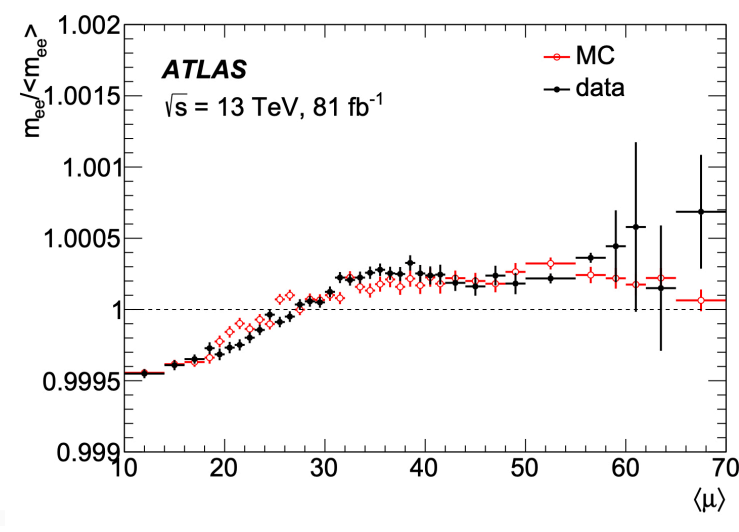

(b)

Figure 6: (a) Residual photon energy scale factors for converted photons from $Z \rightarrow$ ee $\gamma$ and $Z \rightarrow \mu \mu \gamma$ data as a function of the photon transverse energy $E_{\mathrm{T}}$ after calibration with $Z \rightarrow e e$ data. (b) Relative variation of the reconstructed dielectron mass in $Z \rightarrow e e$ events as a function of pileup [7].

\subsection{Timing}

Object timing is calculated online with a resolution of $\sim 1$ ns using OFCs. This online resolution is sufficient for triggering and for vetoing background. Additionally, timing is monitored during the data-taking period for any unusual behavior, and adjustments can be made using so-called fine delays on the FEBs that shift timing in steps of $\sim 100 \mathrm{ps}$.

Offline, timing performance can be significantly improved via calibration to remove timing variations from known effects such as energy and cross-talk dependencies and variations in performance of individual detector components. Using electrons from $W$ and $Z$ boson decays, the resolution can be improved to as low as $\sim 220-270 \mathrm{ps}$ for high-energy electrons and photons, as can be seen in Figure 7. In fact, this level of precision provides the most precise timing in ATLAS and allows LAr timing to be a powerful discriminant for long-lived particle searches [5].

Offline calibration also allows the correlated and uncorrelated component of the timing resolution to be extracted. The correlated component-understood to come from the longitudinal spread of the LHC beam - was computed to be $\sim 200$ ps. Subtracting this in quadrature from the fitted constant term, the uncorrelated contribution attributable to the LAr calorimeter is $\sim 65-185 \mathrm{ps,}$ varying in pseudorapidity and readout gain [5].

\section{Phase I Upgrade}

Run 3, the next data-taking period from 2021 to 2024, presents new challenges that require significant upgrades (known as the Phase I upgrade) to the LAr calorimeter. The LHC will deliver collisions with higher instantaneous luminosity and thus higher pileup. However, ATLAS must maintain the 


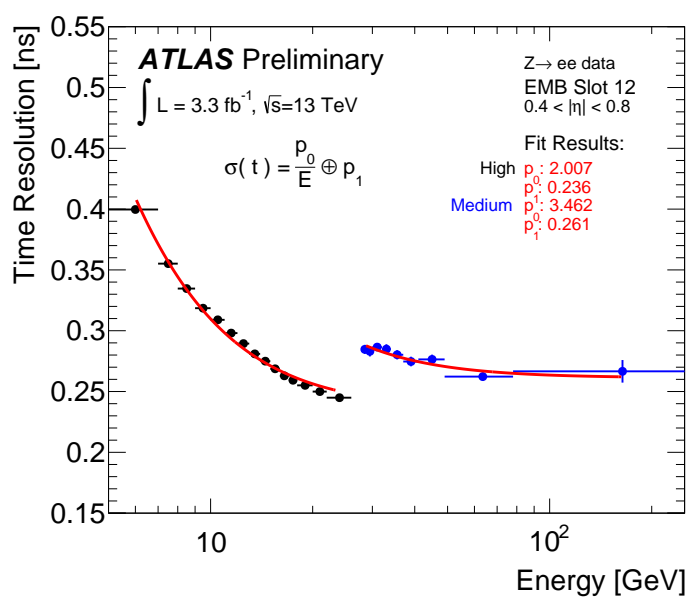

Figure 7: Time resolution after calibration as a function of the energy in the middle layer LAr calorimeter cell with the maximum deposited energy for cells in the barrel with $0.4<|\eta|<0.8$ in 2015. The data is from an independent validation dataset of $Z \rightarrow e e$ events. Fits are to a functional form $\sigma=\frac{p_{0}}{E} \oplus p_{1}$, where $p_{0}$ and $p_{1}$ represent the noise and constant terms, respectively. High and medium readout gains are treated separately [5].

same Level-1 (L1) trigger rates and thresholds. Therefore, better discrimination in the trigger is needed.

The solution is a significant upgrade to the L1 trigger electronics, which will result in a 10 times finer $\mathrm{L} 1$ readout granularity. This will provide improved $\mathrm{L} 1$ discrimination of $e, \gamma, \tau$, and jets based on shower shapes, isolation, etc. It will also improve L1 jet $E_{\mathrm{T}}$ and $E_{\mathrm{T}}^{\text {miss }}$ resolutions. All of these improvements will allow the L1 trigger to handle the increased dominant multi-jet background [8].

Two generations of in-situ demonstrators ran parasitically in the LAr calorimeter barrel throughout Run 2 to validate the design for the upgraded electronics. Good agreement with the main readout was observed, as shown in Figure 8.

\section{Conclusions}

The ATLAS LAr calorimeter operated very successfully throughout LHC Run 2 (2015-2018) with $99.6 \%$ overall data-efficiency. Various Run 2 studies demonstrate the excellent quality of physics data collected and the commitment to continually improving operations and performance. The LAr calorimeter has contributed profoundly to many important physics measurements and searches during Run 2. It provides precise measurements of electromagnetic objects for a wide variety of crucial applications including Higgs boson precision measurements in photonic and leptonic decay modes as well as many searches for physics beyond the Standard Model. It also delivers strong rejection of jet background and measurement of the electromagnetic component of jet backgrounds. As a powerful example of the LAr calorimeter's contribution to Higgs boson precision measurements, the diphoton invariant mass peak is shown in Figure 9, which provides a Higgs boson mass resolution $<0.2 \%$ in the diphoton decay mode alone with only about one fourth 


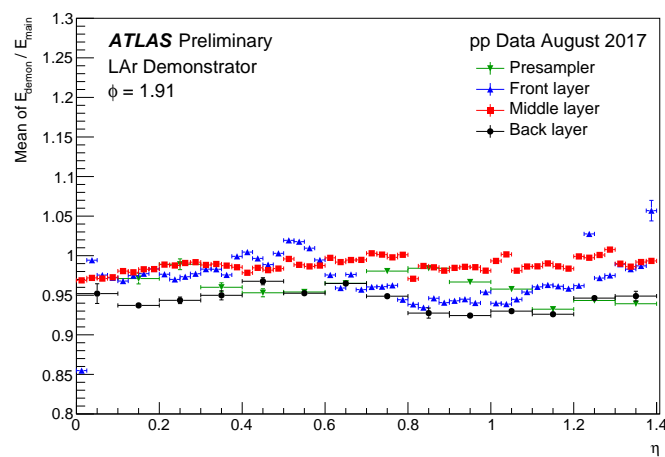

(a)

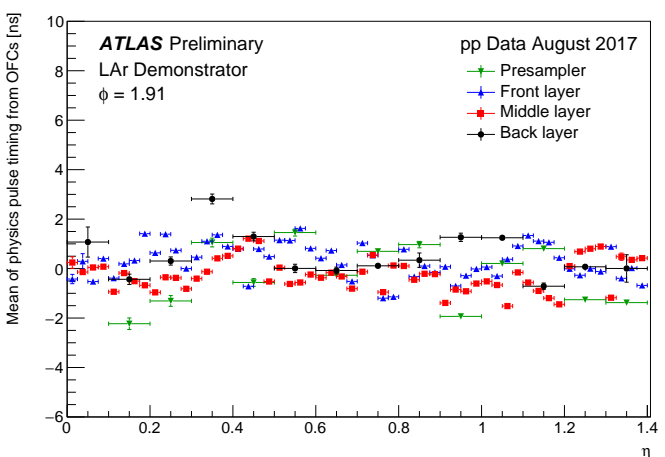

(b)

Figure 8: (a) Ratio of the Phase I readout unit energy to the summed cell energies from the Run 2 main readout as a function of $\eta$ for the slice $\phi=1.91$. (b) Mean value of the Phase I readout unit time measurement. Small shifts of the energy and time are due primarily to the preliminary calibration of the Phase I readout units [9].

of the full Run 2 dataset analyzed thus far. Looking to the future, upgrades for the challenging conditions of Run 3 are well underway, and preparations for further upgrades in Run 4 for the even more challenging conditions of the High Luminosity LHC are also in progress.

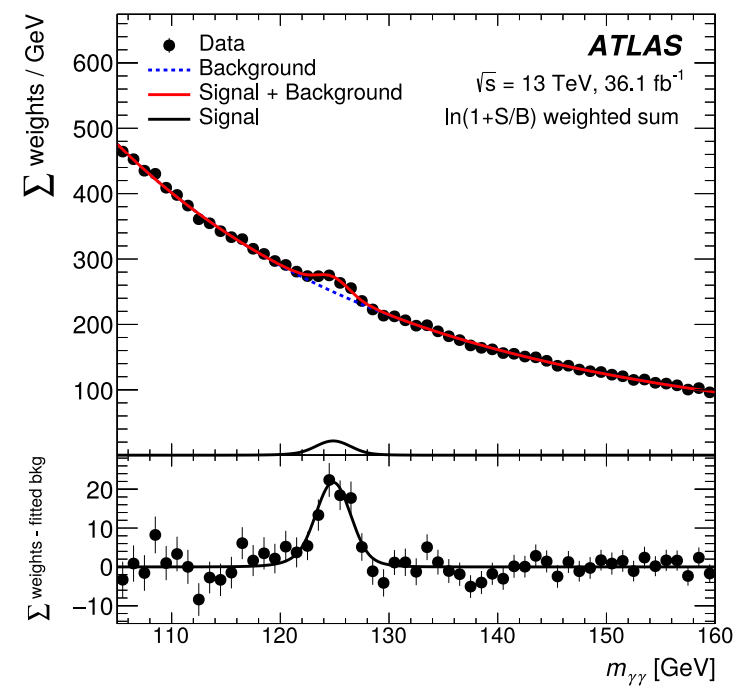

Figure 9: Diphoton invariant mass distribution with $36.1 \mathrm{fb}^{-1}$ of Run 2 data, from which a Higgs boson mass of $m_{H}^{\gamma \gamma}=124.93 \pm 0.40 \mathrm{GeV}$ can be extracted [10].

\section{References}

[1] ATLAS Collaboration, ATLAS Experiment Luminosity Public Results Run 2, https://twiki.cern.ch/twiki/bin/view/AtlasPublic/LuminosityPublicResultsRun2 
[2] ATLAS Collaboration, ATLAS data quality operations and performance for 2015-2018 data-taking, JINST 15 (2019) P04003, arXiv: 1911.04632 [physics.ins-det]

[3] ATLAS Collaboration, ATLAS liquid-argon calorimeter: Technical Design Report, CERN-LHCC-96-041 (1996)

[4] W. Cleland and E. Stern, Signal processing considerations for liquid ionization calorimeters in a high rate environment, Nucl. Instrum. Meth. A 338, no. 2-3, 467-497 (1994)

[5] ATLAS Collaboration, LAr Calorimeter Public Results Run 2, https://twiki.cern.ch/twiki/bin/view/AtlasPublic/LArCaloPublicResults2015

[6] ATLAS Collaboration, LAr Calorimeter Detector Status Public Results, https: //twiki.cern.ch/twiki/bin/view/AtlasPublic/LArCaloPublicResultsDetStatus

[7] ATLAS Collaboration, Electron and photon performance measurements with the ATLAS detector using the 2015-2017 LHC proton-proton collision data, JINST 14 (2019) P12006, arXiv: 1908.00005 [hep-ex]

[8] ATLAS Collaboration, ATLAS Liquid Argon Calorimeter Phase-I Upgrade Technical Design Report, CERN-LHCC-2013-017 (2013)

[9] ATLAS Collaboration, LAr Calorimeter Upgrade Public Results, https://twiki.cern.ch/twiki/bin/view/AtlasPublic/LArCaloPublicResultsUpgrade

[10] ATLAS Collaboration, Measurement of the Higgs boson mass in the $H \rightarrow Z Z^{*} \rightarrow 4 \ell$ and $H \rightarrow \gamma \gamma$ channels with $\sqrt{s}=13 \mathrm{TeV}$ pp collisions using the ATLAS detector, Phys. Lett. B 784, 345-366 (2018), arXiv: 1806.00242 [hep-ex] 